\title{
Rechts-Bewegungs-Kunst für das 21. Jahrhundert. Rudolf Wiethölter zum 80. Geburtstag1
}

\author{
„Freiheiten unter Auflagen - ein Projekt, \\ das Verbote in Versuchungen verstrickt wie \\ Versuchungen in Verbote. Das ist \\ Kontingenzbewältigungspraxis \\ einer 'kunstvollen' Theorie in being. " 2
}

\section{Von der Entzauberung des Rechts zur produktiven Utopie}

Mit seiner „Rechtswissenschaft ${ }^{\text {“ } 3}$ schreibt sich Rudolf Wiethölter im bewegten Jahr 1968 in die Geschichte der Jurisprudenz ein. Entzauberung des Rechts markiert die Stoßrichtung seiner politischen Rechtstheorie. Deren offene Epistemologie und Logik erweist sich alsbald als der unablässige Versuch, das magische Dreieck einzukreisen, das von den großen Theorielagern - der kritischen (Diskurs-)Theorie des Rechts, der ökonomischen Rechtsanalyse und der Systemtheorie - abgesteckt wird. Schlüsselbegriffe dieses anspruchsvollen Unternehmens sind die „Recht-Fertigung“, die in das Recht eingelassenen Paradoxien und die daraus entwickelte Konzeption eines prozeduralisierten Kollisionsrechts, das erstens zwischen Recht und Nicht-Recht, d.h. zwischen Recht und den Funktionsimperativen anderer gesellschaftlicher, individueller und natürlicher Um-, Mit- und Nachwelten vermittelt, ${ }^{4}$ - im Anklang an die Systemtheorie - und dabei zweitens von "Skepsis-Verheißungen“, einer „produktiven Utopie“ nach Art der kritischen Theorie, geleitet wird. Wiethölter geht es darum, den Eigensinn solcher Autonomiebereiche durch Recht zu einem neuen Gemeinsinn zusammenzubringen. Heute heißt das, die Forderung nach justice, prudence, benevolence an eine gesellschaftliche Netzorganisationslogik zu richten. Im Vordergrund steht damit der kritisch-politische Bewegungsaspekt der nach wie vor politischen Rechtstheorie.

Um diesem gerecht zu werden, muss man Wiethölters Projekt zunächst an jene historisch-gesellschaftlichen Entwicklungsdynamiken zurückbinden, die sich seit Ende der 60er, Anfang der 70er Jahre in ihr Gegenteil verkehrt haben, und erst neuerdings wieder, mit dem Aufbrechen einer sich als Finanzkrise präsentierenden neuen kapitalistischen Akkumulationskrise unter kritischen Veränderungsdruck zu geraten scheinen. ${ }^{5}$ Damals leidet der organisierte Industrie-Ka-

1 Ich danke Andreas Fischer-Lescano und Günter Frankenberg für hilfreiche Anregungen.

2 Vgl. R. Wiethölter, Rechtfertigungen eines Gesellschaftsrechts, in: C. Joerges/G. Teubner (Hrsg.), Rechtsverfassungsrecht, Rechtfertigungen zwischen Privatrechtsdogmatik und Gesellschaftstheorie, Baden Baden 2003, S. $13 \mathrm{ff} .(21)$.

3 Vgl. R. Wiethölter, Rechtswissenschaft, Fischer Funkkolleg, Frankfurt am Main 1968.

4 Vgl. dazu A. Fischer-Lescano/G. Teubner, Prozedurale Rechtstheorie: Wiethölter, in: S. Buckel/R. Christensen/A. Fischer-Lescano (Hrsg.), Neue Theorien des Rechts, 2. Auflage, Stuttgart 2008. Siehe auch F. Steinhauer, Geflügelte Worte. Zum Achtzigsten des Juristen Rudolf Wiethölter, in: Frankfurter Allgemeine Zeitung Nr. 161 vom 15.7.2009, S. N 5.

5 Vgl. zu den Kritikformen der 68er Bewegungen und ihrer selektiven Internalisierung durch den FinanzKapitalismus L. Boltanski/E. Chiapello, Der neue Geist des Kapitalismus, Konstanz 2003, S. 213 ff.; C. Marazzi, The Violence of Financial Capitalism, Semiotext, Los Angeles 2009. 
pitalismus an einem Wachstums- und Rentabilitätsrückgang, der mit der Verlangsamung der Produktivitäts-Gewinne als Folge stetig steigender Reallöhne und sozialer Absicherungs-Errungenschaften zusammenhängt. Die Kritik steht demgegenüber in ihrem Zenith, wie die Ereignisse von 1968 belegen, als eine marxistisch geprägte Sozialkritik und lebensweltliche Emanzipationsforderungen einen Schulterschluss eingehen. Stichworte: Befreiung des autonomen, authentisch-kreativen Potenzials des Einzelnen qua Emanzipation von staatlicher Bevormundung, von kapitalistischer Zurichtung und Ausbeutung und von bürgerlicher Moral sowie die entsprechende Entwicklung neuer Formen von sozialer Solidarität.

In den letzten drei Jahrzehnten hat sich diese Konstellation im Sinne einer kapitalistischen Neuordnung verändert. Stichworte: finanzlogische Überformung der Produktion von Gütern und Dienstleistungen und Wandel der Beziehungen zwischen Kapital und Arbeit (Flexibilisierung). Privatisierungs- und Deregulierungsinitiativen unterwerfen die Regierungen, den 'öffentlichen Sektor' (last not least: die Universitäten) und die Gesellschaft insgesamt den Kriterien von Unternehmens-Management und wirtschaftlicher Effizienz. Die Gesellschaft beobachtet ihre Vernetzungen von wirtschaftlichen, politischen, rechtlichen, wissenschaftlichen usw. Funktions-'Regimes' als globale 'Risiko-Gesellschaft', und das flexible, kreative 'unternehmerische Selbst' wird zur neuen Subjektivierungsform. ${ }^{6}$ In allen Sach-Zusammenhägen und auf allen (Funktions-)Ebenen kollidieren nunmehr neue Formen des Regierens (oder der Governance) mit traditionellen Konzepten der Regierung, die sich noch entlang der Unterscheidung von Öffentlichem und Privatem bewegen.

Damit gelangt aber das Recht 'zurück' zu seinem historisch-normativen Produktions-Potenzial, zur Wiethölter'schen „Recht-Fertigung“. Hier kommt Rudolf Wiethölters Ansatz praktisch zu sich selbst und enthüllt seine Liaison mit der historisch-gesellschaftlichen Praxis. Es zeigt sich, dass das Recht nicht an ein spezifisches Polis-Modell oder einen hobbesschen Staat gebunden ist. Das Recht ist in der Lage, verschiedene Formen des Gemeinwesens (mit) zu gestalten und zu gewährleisten, solange es damit seiner besonderen normativen MöglichkeitsBedingung, seinem Anspruch entspricht, den Überschuss an gerechteren Gestaltungs-Möglichkeiten des Gemeinwesens zum Maßstab seiner Konstruktionen zu machen.

Sein „Unbehagen am Recht“ fasst Wiethölter 1968 wie folgt zusammen: „Das Unbehagen am Recht ist folglich Unbehagen daran, dass wir Recht suchen, weil wir es brauchen, es aber für uns und unsere Zeit nicht finden können, weil es noch nicht da ist". 7 Das klingt wie Valentinsches Spruchgut für verlorene/verratene Revolutionen: „Mögen täten wir schon wollen, aber dürfen haben wir uns nicht getraut“. Aber Wiethölter traut sich und wird mit seinem Vertrauen in die Kraft politisch-sozialer De- und Rekonstruktion des Rechts zu einem Protagonisten eben jener Zeit, in der diese 'Haltung' zum Leitmotiv und zum Zentrum sozialer Bewegungen wird. Sein kritisch-theoretisches, ökonomisch informiertes und praktisch-politisches Programm findet sich in a nutshell (natürlich!) dort,

6 Vgl. U. Bröckling, Das unternehmerische Selbst - Soziologie einer Subjektivierungsform, Frankfurt am Main 2007. Zu den neuartigen 'glokalen' Konfliktrisiken und entsprechenden (rechtlichen) Lösungsangeboten siehe A. Fischer-Lescano/G. Teubner, Regimekollisionen, Zur Fragmentierung des globalen Rechts, Frankfurt am Main 2007; und C. Joerges/F. Rödl, Zum Funktionswandel des Kollisionsrechts II, Die kollisionsrechtliche Form einer legitimen Verfassung der post-nationalen Konstellation, in: G.-P. Calliess/A. Fischer-Lescano/D. Wielsch/P. Zumbansen (Hrsg.), Soziologische Jurisprudenz. Festschrift für Gunther Teubner zum 65. Geburtstag, Berlin 2009, S. 765 ff.

7 Vgl. Wiethölter (Fn. 3), S. 41. 
wo man es nicht erwartet: in einem Diskussionsbeitrag zum 1970er Referentenentwurf eines $\mathrm{GmbH}-G e s e t z e s .{ }^{8}$ Dort schreibt er:

Befund: „Unser Recht ist hoffnungslos rückständig. Wir leben noch im bürgerlichen Rechtsstaat des 19. Jahrhunderts mit Institutionenstaat und Formenrecht. Der demokratische Sozialstaat heute zielt auf eine politische Gesellschaft mit Funktionenrecht." Das institutionelle Setting des Gesetzgebungsverfahrens erweist sich als undemokratisch, angefangen bei den geheimen Beratungen und der selektiven Rekrutierung von Sachverständigenkommissionen und der „Neutralisierung rivalisierender Durchsetzungsansprüche“ im Wege der „Technik-Reduktion“: „Das Parlament registriert verantwortlich, aber programmiert nicht mehr, die Bürokratie programmiert unkontrolliert, aber verantwortet nicht, die Regierung vermittelt ihre Prioritäten jeweiliger Interessen-Einflüsse; die Sachverständigen (an der Spitze die 'unabängigen Wissenschaftler'!) vom Dienst werden instrumentalisiert.“ Die gesellschaftliche Öffentlichkeit wird auf „Loyalitäts-Akklamationen“ reduziert. Dabei reproduziert die „Verbundkooperation“ von organisiertem Privatkapitalismus und staatlicher Rahmengewährleistung die „klassische Problematik der bürgerlich-kapitalistischen Gesellschaft: gesellschaftliche Produktion - private Aneignung. “

Therapie: „Angesichts der gesellschaftlichen Grundstrukturen (Systemintegration über Krisen- und Konjunkturmanagement), die sich nicht spalten lassen in 'Staat' und 'Gesellschaft' (darin dann u.a. 'Wirtschaft'), ist theoretisch wie praktisch nur noch für einen Arbeitsansatz Platz: für die sog. 'Sozialwissenschaft' ('Systemtheorie').“ Dieselbe präsentiert sich ihm damals in drei „Orientierungen“: normativ-teleologisch, empirisch-analytisch und historisch-dialektisch. Er nimmt Partei für die dritte Orientierung, nach der „die konkrete Gesellschaft (...) strukturell-emanzipativ ihre mögliche Praxis (bestimmt)“, und erklärt: „Ich mute niemandem zu, sich dem Lager 3 anzuschließen, in dem ich arbeite. Ich muß aber jedem zumuten zu erkennen, daß er mit der Entscheidung für das Lager 1 irrational-dezisionistisch ('gläubig'), restaurativ-konservativ ('das gute Alte und Wahre') Partei genommen hat.“ Hingegen „(orientiert sich) die moderne sozialwissenschaftliche Systemtheorie' (...) nicht an vorgegebenen normativen 'Ordnungen' und 'Institutionen', sondern an registrierbaren sozialen Handlungen und Handlungs-(System-)Zusammenhängen. Sie zielt auf den sozialen Prozess, nicht auf gedankliche Abstraktionen oder ontologisierte Wertewelten. "Entsprechend wirkt das Recht als „Teil politisch-sozialer Theorie und politisch-sozialer Praxis. Alle Rechtsentscheidung (von der 'Rechtsgebung' bis zur 'Rechts-Anwendung'; der Dualismus ist selbst obsolet geworden!) wird zur Handlungs(Entscheidungs-)Orientierung an der politisch-sozialen 'Erkenntnis' unserer Gesellschaft selbst. Dass solche Einsicht nicht Allgemeingut wird, hat ihrerseits Funktion: die Einsicht würde, ziehen wir die notwendigen Folgerungen, unser Rechtsverständnis nicht weniger als unsere gesellschaftliche Organisation 'revolutionieren'. Gegen diese Einsicht aber ist das 'System' integrativ abgedichtet.“ Diese Abdichtung erfolgt, so Wiethölter, „über Bewußtseinsindustrien und Ausbildungssysteme nahezu unmerklich“. „Wissenschaften und Universitäten in ihrer angeblich unpolitisch arbeitsteiligen Verwendungswirkung“ spielen dabei mithin eine entscheidende Rolle. Folglich „(tritt) die Bedeutung von Hochschulund Studienreform als derzeit politisch gefährlichster Kritik der Gesellschaft (...)

8 Siehe zum Folgenden R. Wiethölter, Die GmbH in einem modernen Gesellschaftsrecht und der Referentenentwurf eines GmbH-Gesetzes, in: Probleme der GmbH-Reform, Köln 1970, S. 18 ff.

9 Es handelt sich hier nicht um die Parson'sche bzw. frühe Systemtheorie Niklas Luhmanns, allerdings um eine kritische Theorie der Gesellschaft 'als solcher', die die gesamte Gesellschaft durchdringenden Struktur-Bildungen und -Entwicklungen zum Gegenstand hat. 
zutage." Kein Zufall also, dass er sich auf diese Reform konzentriert und ein Konzept für die Juristenausbildung entwirft, welches das Recht als Sozialwissenschaft erfahrbar macht und jene politisch-sozialen Einsichten vermittelt, die angemessene(re) Strukturbildungen für eine komplexe emanzipierte politische Gesellschaft ermöglichen.

Rudolf Wiethölter verfolgt diese Reform sowohl mit seinem Zugang zur institutionellen Politik - Einführung einer einphasigen Juristenausbildung mittels Landesgesetzgebung - als auch mit dem Einsatz der ihm zur Verfügung stehenden materiell-personellen Infrastruktur, und d.h. mit dem 'Institut für Wirtschaftsrecht', das sich bis zum Umzug der Fakultät im Herbst 2008 im 3. Stock des Frankfurter Juridicums in der Frankfurter Senckenberganlage 31 befindet. ${ }^{10}$ Dabei geht es ihm nicht zuletzt um eine indirekte Reduktion des Einflusses von Funktionseliten, die mittels ihrer „juristischen Subkulturen“ („gebildete Oberprofessoren“ und „gelehrte Oberrichter“) „die Gesellschaft in Schach halten “ und über Rechts-Fakultäten und (Ausbildungs-)Gerichte dazu beitragen, die Rolle des universitären (Rechts-)Studiums als (selbst-)kritisches 'psycho-soziales Moratorium' (Erik Eriksson) zu boykottieren.

Einphasenmodelle werden in verschiedenen Bundesländern aufgelegt. Während ein kritisches Juristenausbildungsmodell Wiethölter'scher Provenienz 1972 mit der Fakultätsgründung in Bremen als Modellversuch realisiert werden kann, scheitert ein solches 1974 in Hessen. Zumindest Anteile davon lassen sich mit der Einführung eines sozialwissenschaftlichen 'Grundlagenscheins' retten sowie in Form eines Forschungsprojekts 'Studium und Praxis', das unter der Federführung des Hessischen Justizministeriums steht und an dem Wiethölter sich von 1977 bis 1981 mit dem Unterprojekt 'Juristische Methode und Rechtsdogmatik als Juristenpraxis' beteiligt.

Um so wichtiger wird der '3. Stock', in dem Wiethölter sein theoretisches RechtsBeobachtungs-Konzept als Studiums-, Forschungs- und praktisch-politisches Transformations-Modell institutionalisieren kann. Dazu gehören folgerichtig auch Transformationen im eigenen Haus, wie pathetisch einigen Zeitgenossen dieses 'Wagen von Demokratie' auch anmuten mag: Assistentenstellen werden gepoolt, um möglichst vielen interessierten Studenten ein unabhängiges Studium zu gestatten und das vormoderne akademische Adeptentum auszuhebeln. Die Abschaffung der Habilitation als dritter universitärer Subkultur-Bewährungsprobe gelingt zumindest im Bremer Modellversuch. Es geht um die demokratisch organisierte gemeinsame Einzelproduktion kollektiver Intelligenz. Dass viele seiner Studenten Wiethölter gerade wegen dieser Haltung bewundern, erweist sich als unvermeidliche Nebenwirkung eines gelungenen Umgangs mit dem alteuropäischen Paradox der Erziebung zur Freibeit.

Um den Anforderungen und Zumutungen des politischen Rechts gerecht zu werden, wird die Juristenausbildung im Stile des 3. Stocks genau zu dieser paradoxen Angelegenheit, einem permanenten Münchhausen-Akt. Es geht darum, „zu begreifen, was aus 'Gott und seinem Recht' geworden ist, säkularisiert bis auf die Knochen und doch so verheißungsbedürftig wie am ersten Tag." Dazu bedarf es eingehender Studien im Stile Michel Foucaults zu Architektur und Auswirkungen der historischen Gesellschafts-Rechts-Form. Man muss herausfinden, wie diese mit der riskanten Reproduktion autonomer Einzel-Rationalitäten umgeht; wie Konflikt-Kollisionen politisch, rechtlich, wirtschaftlich usw.

10 Seit 1998 ist G. Teubner am 3. Stock Wiethölters Nachfolger. Nichtsdestotrotz laufen die Wiethölter'schen Seminare dort weiter; und dies auch nach dem Umzug auf den Campus Westend. Vgl. zur Veranstaltung im WS 2009-2010: http://www.jura.uni-frankfurt.de/ifawz1/teubner/Mittwochsseminar/index.html. 
gehandhabt werden; welche Legitimations-, Kontroll- und ImmunisierungsMechanismen installiert werden, um dieses 'Netzwerk' gegen Zerfall und Widerstand zu verteidigen und gleichzeitig ausreichende Offenheit zugunsten des für die Selbstreproduktion notwendigen Wandels zu gestatten.

Dementsprechend müssen sich Studenten einerseits mit dogmatischen Konzepten und Methoden vertraut machen, die sich bei der Konstruktion und Lösung solcher Kollisionen bewährt haben. Um die einschlägigen Leitunterscheidungen in Relation zu anderen Möglichkeiten 'begreifen' zu können, müssen sie sich sodann mit einer Beobachtungsform anfreunden, die die aktuellen Standards für die Behandlung von Normen und Tatsachen jeweils suspendiert und eine Analyse des einschlägigen Regelungskontexts erlaubt. Angesichts des Reflexionslocus '3. Stock' kann es sich dabei natürlich nur um die Institutionalisierung von (Selbst- und Fremd-)Beobachtungen (mindestens) '3. Ordnung' handeln: ${ }^{11}$ Die herrschenden Rechtsunterscheidungen werden zunächst mit der Rolle verglichen, die die Sozialtheorien mit der größten Wirkungsmacht dem Recht in ihrem jeweiligen Gesellschafts-Konzept einräumen. Als solche Theorien kristallisieren sich für Wiethölter, wie gesagt, im Laufe der Zeit Systemsoziologie, Politische Ökonomie und Kritische Philosophie heraus. Dabei zeigt sich, dass sie, ebenso wie alle Beobachtungen, mit ihrem eigenen 'blinden Fleck' zu rechnen haben: Ihre Unterscheidungsbildung ist in ein komplexes Geflecht von Voraussetzungen und Auswirkungen eingelagert und kommt nicht ohne „dezisionistischen Rest" (Habermas) aus.

Das heißt aber auch, dass selbst dann, wenn keine von ihnen vom Recht viel übrig lässt, erst die eigentliche rechtliche Rekonstruktionsarbeit, das rechtliche Proprium, beginnt. Von 'großen Erzählungen' ist mithin auf dem 3. Stock schon lange vor dem Einläuten der sog. 'Postmoderne ${ }^{12}$ nicht mehr die Rede. Die untersuchten Sozialtheorien werden einerseits angesichts ihrer Gestaltungskraft und ihrer Rechts-'Irritationen' ernst genommen, aber auch auf ihre Möglichkeitsbedingungen und Begründungsgrenzen hin untersucht. Dieser Reflexionsprozess fördert - wie es im Anschluss an die Beobachtungslogik der Systemtheorie heißt - ihre Kontingenz zu Tage. Ihre Beschreibungen sind, ebenso wie die des Rechts selbst, weder notwendig noch schicksalhaft vorherbestimmt. Sie sind stets auf andere Weise möglich und verlieren daher nie ihre Unangemessenheit.

Damit stellt sich aber zugleich die historisch-politische wie auch normative Frage, wie soziale Organisation und ihre politischen, wirtschaftlichen, rechtlichen, wissenschaftlichen usw. Funktionen sich verändern müssen, um negative Auswirkungen zu verhindern und das demokratische Gemeinwesen, Gemeinwohl, Gerechtigkeit, Wahrheit, usw. angemessener zu gestalten. Hier sind wir folglich mit Rudolf Wiethölter bei der Verheißung angelangt: der rechtlichen Gewährleistung einer freien Verwirklichung autonomer Entwicklungschancen unter Einbau sozialer Rücksichtnahmen/Reziprozitäten. Er nennt dies das „bürgerlich wie anti-bürgerlich unerledigte nach-feudalistische Sachprojekt“.

Die erwähnten Sozialmodelle werden von Wiethölter also dazu benutzt, eine gerechte(re) Definition des Gemeinwohls oder der Reziprozität für den besagten Kontext zu entwerfen. Dies geschieht auf der Basis entscheidungsleitender rechtlicher Machbarkeits-Gutachten: Er betont, es gehe ihm „eher um die einlösbaren Ermöglichungs- als die uneingelösten Verheißungs-Überschüsse“. ${ }^{13}$ Die Eigen-

11 Vgl. zu dieser entparadoxierenden (Selbst-)Reflexions-Hierarchie im Sinne neokonstruktivistischer Theorien N. Luhmann, Die Gesellschaft der Gesellschaft, Frankfurt am Main 1997, S. 1109 ff.

12 Dafür steht bekanntlich die Arbeit von J.-F. Lyotard, Das post-moderne Wissen, Wien 1982.

13 Vgl. Wiethölter (Fn. 2), S. 18. 
werte der in Streit stehenden autonomen Funktionsbereiche, wie etwa der Wirtschaft oder des Rechts, werden vom ihm grundsätzlich anerkannt, um sie sodann als Freibeits-Modalitäten zu verklammern und dementsprechend das Risiko/die Verantwortung für das (Nicht-)Eintreten erwarteter Folgen auf die Konfliktparteien zu verteilen.

Die Ausbildung in solchen theoriegesteuerten Recherchen zum einschlägigen sozialen Netzwerk und in der entsprechenden rechtspolitischen (Folgen-)Beurteilung ist genau das, was das traditionelle, autoritäre Rechtsdenken vermeiden will: „Juristen müssen ihre Methoden haben, aber sie dürfen nicht über sie verfügen können“, heißt es bei Ernst Forsthoff. Entscheidungen darüber, wie die Gesellschaft zu organisieren ist, sind anderswo zu treffen, und Juristen haben solche Entscheidungen umzusetzen, ohne ihre Methoden für autonome 'Steuerungsversuche' zu benutzen. Hingegen verheißt Wiethölter:

„Vielleicht liegt in der Emanzipation solchen Rechts vom Recht in den rivalisierenden Gesellschaftstheorien (...) ein Verwirklichungs-Chancenschritt. 'Recht' beugte sich dann nicht den Gesellschaftstheorie-Designs, sondern wäre selbst eines, mithin jedenfalls nicht 'System', nicht 'Diskurs,' nicht 'Unternehmen'."14

Oder im Sinne einer seiner Lieblingsparabeln: „Im Hase-Igel-Spiel zwischen Juristen, Soziologen und vielen Anderen können/möchten schließlich auch Juristen einmal Igel sein.“

\section{Kritik der Kritik}

Der von Wiethölter im 3. Stock institutionalisierte Erkenntnis- und praktischpolitische Transformations-Prozess verfolgt mithin ein ubiquitär einsetzbares, unmittelbar politisch wirksames Rechts-Entdeckungs- und Methodenprogramm. Es entspricht dem, was Foucault ${ }^{15}$ als das zentrale Ethos der Aufklärung identifiziert hat: Die 'Befreiung des Möglichen' durch das sich fortlaufend erneuernde Kontingenzbewusstsein; 'das Andere' in Kant, eine kritische Ontologie, die alle apriorischen Vernunftbedingungen letztlich torpediert. „Alles Mögliche ist möglich!“ Diese 'Kritik der Kritik' bringt gemeinsam mit ihrem (paradox)ontologischen Aspekt einen permanenten normativen Druck auf die unvermeidlichen Asymmetrien rechtlich vermittelter gesellschaftlicher Organisation hervor, auf ihre Mechanismen von Inklusion und Exklusion, Disziplin und Kontrolle, Eigentum und Knappheit usw., die das tendenziell unbegrenzte Entwicklungspotential individueller und sozialer Akteure selektiv einhegen und ausnutzen.

Damit ist das Wiethöltersche Methodenprogramm gleichzeitig poietisch-unsystemisch angelegt: „Geschichte (Recht usw.) ist nicht gegeben, sondern aufgegeben“, lautet einer seiner vielen Lehrsätze, die in der Regel der deutschen 'Dichtung und Wahrheit' entstammen, jedoch gleichzeitig an die das Begreifen stimulierende Verblüffungswirkung fernöstlicher Zen-Koans erinnern: ${ }^{16}$ 'Aufgegeben' jedenfalls als produktiv-kreative (poietische) Strukturierungs-Last/-Lust, die als Rechts-Kunst (frei nach Adorno) „Chaos in Ordnung bringen soll“; und 'aufgegeben' im Sinne der vom Recht versprochenen Dauerveränderung solcher selektiver historischer Strukturen zur 'Bewältigung' ihrer unvermeidlichen (systemischen) Unangemessenheit.

15 M. Foucault, Was ist Aufklärung?, in: E. Erdmann/R. Forst/A. Honneth (Hrsg.), Ethos der Moderne. Foucaults Kritik der Aufklärung, Frankfurt am Main 1990.

16 Originalton Wiethölter: „Ich selbst habe mehr als nur 'sympathy' für Erörterungen nach Art des alten Fußballzauberspiels von Schalke 04 oder der Filme Alexander Kluges.“. 
Wiethölters Ansatz wirkt damit jenseits der Funktionsweisen der offiziellen Politik politisch und erweist seine Affinität zu Zielen und Formen sozialer Bewegungen, ${ }^{17}$ allerdings nur insofern diese tendenziell das selbstkritische Kontingenzbewusstsein auf sich selbst anwenden und bei ihren Vorhaben mitlaufen lassen. Die Einsicht in die eigene Begrenztheit und Imperfektion resultiert in einem neuen Konfrontations-Stil, einer „Streitkultur“, praktisch einem Kampf mit gebremstem Schaum, vor allem ohne den Gegner beseitigen zu wollen. Das Recht hat diese praktische 'Haltung' zu vertreten und in seinen Verfahren zu gewährleisten. Aber die Wirkung politischer Aktionen, sei es der klassischen Arbeiterbewegung als auch der New Globals, speist sich doch gerade aus der in kämpferischer Form vorgetragenen Systemkritik und Empörung angesichts der von der Übermacht gesellschaftlicher Immunisierungsstrukturen nicht realisierten, aber als realisierbar erachteten emanzipativen Überschüsse?! Pflege und Behandlung dieser neuerlichen Paradoxie finden sich, als erstem Schritt, in der Erweiterung und rechtlichen Anerkennung des Begriffs der 'Sozialen Bewegung': Er steht für die Gesamtheit und Dynamik des auf vielen sozialen Ebenen einzeln und kollektiv vorgetragenen kritisch-normativen Drucks auf existierende gesellschaftliche Asymmetrien/Benachteiligungen. ${ }^{18}$ Die 'Benachteiligten' haben mithin das Recht(!), jene Partizipations-Verheißungen, aus denen das Recht selbst seine Legitimation zieht, zu realisieren.

In einem zweiten Schritt muss man eine Ebenen-Verschleifung vornehmen: Der Sturm auf die Bastille und seine Abkömmlinge nehmen an Recht-Fertigung zu, je mehr der auf (funktional) organisierten sozialen Ebenen ausgetragene politische Kampf um das „Andere“ existierender politischer, wirtschaftlicher, rechtlicher usw. Ansätze an Kraft verliert. Dieser Balanceakt weist seinerseits wieder auf die konkrete Utopie der Wiethölter'schen Rechts-Bewegungs-Kunst zurück: eben auf die als 'Prozeduralisierung' bezeichnete verbindliche, situative Festlegung von Kollisions-Regeln in Foren und Verfahren, die es nicht zum Äußersten kommen lassen. Das „Projekt Freibeiten unter Auflagen“ nimmt hier also eine unerwartete subversive Wendung: Je weniger autonome, soziale Regimes sich auf horizontale Verhandlungs- und Deliberationsprozesse in einer Streitkultur einlassen und stattdessen etwa auf ihre (ökonomische, politische usw.) Durchsetzungsmacht setzen, desto weniger stehen auch außerorganisatorische (spontane) Formen sozialer Bewegungen unter den Auflagen der Streitkultur.

Die Aktualität und Dringlichkeit des Wiethölter'schen Projekts zeigen sich hier in aller Deutlichkeit; ebenso seine Verwandtschaft zu dem 'Polis-Konzept', das Boltanski und Chiapello für die kapitalistische Netzwerk-Gesellschaft des 21. Jahrhunderts vorgeschlagen haben: ${ }^{19}$ eine „Projekt basierte Polis“, die die Grundbedingungen des Netzwerks - die potentiell totale Verbindung zwischen allen 'Knoten', seine Polyzentrizität und seine grundsätzliche Heterarchie, seinen Ereignischarakter und den permanenten Fluss zahlloser Kontakte - mit den Anforderungen einer legitimen sozialen Organisation und Regulierung in Einklang bringen soll. 'Projekte' sind dann unzählige, auf dem nahtlosen Gewebe des Netzes eingerichtete Wert schaffende Akkumulationsräume, deren Strukturen der Legitimation bedürfen. Das 'Projekt Freiheiten unter Auflagen`zielt treffsi-

18 Siehe dazu G. Frankenberg, National, Supranational, Global: Ambivalenzen zivilgesellschaftlicher Praxis, in: M. Blecher/G. Bronzini/J. Hendry/C. Joerges (ed.), Governance, Civil Society and Social Movements, European Journal of Legal Studies, Vol. I, Nr. 3, 2008, pp. 1-32; Online-Veröffentlichung unter $<$ www.ejls.eu>.

S.o. Fn. 5. 
cher auf die Lösung dieser Form sozialer Raumgestaltung. Resultat der rechtlichen Verfassung unzähliger solcher Akkumulationsräume ist ein kontextualisiertes Gemeinwohl. Andererseits werden die Gerechtigkeits-Forderungen vom Recht wie von sozialen Bewegungen - stets auch an das Netzwerk als solches herangetragen. Wiethölter hat beide Aspekte im Sinn, wenn er „eher die einlösbaren Ermöglichungsüberschüsse“, d.h. konkret machbare Alternativen, anvisiert, aber die „uneingelösten Verheißungs-Überschüsse“ stets mitlaufen lässt: „Kunstvolle“ Kritische Rechtstheorie „in being“, das ist die demokratisch vernetzte Realisierung sozialer Maßstabsbildung unter Dauer-Verweisung auf angemessenere Möglichkeitsüberschüsse, in denen sich stets auch das 'generalisierte Andere' der existierenden sozialen Ordnung erhält. Rudolf Wiethölters konstruktiver Rechts-Bewegungs-Kunst gelingt es mithin, in Abwandlung des berühmten Adornoschen Diktums, ihre Verwirklichungen und Versäumnisse als Recht-Fertigungs-Ereignis dauerhaft zu verschränken.

\section{Demokratische und soziale Gesellschaftsverfassung in Europa}

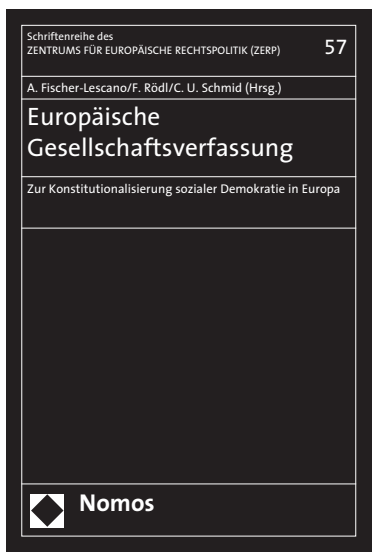

\section{Europäische \\ Gesellschaftsverfassung}

Zur Konstitutionalisierung sozialer Demokratie in Europa Herausgegeben von Prof. Dr. Andreas Fischer-Lescano, Dr. Florian Rödl und Prof. Dr. Christoph Ulrich Schmid 2009, 408 S., brosch., 98,-€, ISBN 978-3-8329-5087-3 (Schriftenreihe des Zentrums für Europäische Rechtspolitik der Universität Bremen (ZERP), Bd. 57)

Der Band betrachtet das Privat-, Wirtschafts- und Arbeitsrecht als Gegenstand des europäischen Verfassungsrechts. Die Beiträge stellen aktuelle rechtspolitische Auseinandersetzungen in ihrem gesellschaftlichen Kontext dar. 